\title{
UN ESTUDIO FENOMENOLÓGICO ACERCA DE LAS PERCEPCIONES DE LOS PROFESIONALES DE ENFERMERÍA SOBRE LA MUERTE
}

\section{A PHENOMENOLOGICAL STUDY ABOUT THE PERCEPTIONS OF NURSING PROFESSIONALS TO DEATH}

Rosalia Cara Rodríguez

Servicio Andaluz de Salud

Cara Rodríguez, R. (2020). UN ESTUdio FENOMENOLÓGICO ACERCA DE LAS PERCEPCIONES DE LOS PROFESIONALES DE ENFERMERÍA ANTE LA MUERTE. Revista Ene De Enfermería, 14(1). Consultado de http://www.ene-enfermeria.org/ojs/index.php/ENE/article/view/961 


\section{Resumen}

La muerte y su afrontación se consideran socialmente como un tema tabú. El objetivo de este estudio es analizar y describir las experiencias de los profesionales de Enfermería respecto a la muerte en el ámbito personal y laboral. El método utilizado ha sido la investigación cualitativa basada en la fenomenología hermenéutica con marco epistemológico y metodológico de Gadamer, donde la recogida de información se realizó con una entrevista grupal focal a los profesionales de Enfermería. Como conclusiones finales se obtuvieron: la educación al personal de Enfermería sobre cómo afrontar la muerte de los pacientes es una medida de actuación necesaria para conseguir mantener una adecuada calidad de vida laboral.

Palabras clave: muerte; tabú; personal de Enfermería; lugar de trabajo; humanos.
Abstract

Death and its confrontation are considered socially as a taboo subject. The objective of this study is to analyze and describe the experiences of nursing professionals regarding death in the personal and work environment. The method used was qualitative research based on hermeneutical phenomenology with an epistemological and methodological framework by Gadamer, where the collection of information was carried out with a focal group interview with nursing professionals. The final conclusions were: education of nursing staff on how to deal with the death of patients is a necessary action measure to maintain an adequate quality of working life.

Keywords: death; taboo; nursing staff; workplace; humans. 


\section{INTRODUCCIÓN}

El concepto de la muerte y su proceso en las sociedades occidentales siguen siendo un tema tabú. La percepción de actuación profesional del personal sanitario sobre el tema de la muerte no es ajena a las creencias y prácticas sociales donde desarrollan su labor (1).

La muerte es un proceso de la vida que afecta a todos los seres humanos. Los cuidados paliativos tienen como objetivo principal aliviar el sufrimiento y mejorar la calidad de los pacientes en etapas finales de la vida. Esto supone que las Administraciones sanitarias y los profesionales deben asumir el reto de prestar una atención integral no solo a los aspectos emocionales, físicos, espirituales y sociales, sino también de asegurar que dicha atención se preste en un lugar idóneo (2).

Enfermería debe otorgar al paciente paliativo el papel de ser partícipe de sus cuidados y su atención, estableciendo así una relación de ayuda(3), además de brindar al paciente y a su familia la capacidad de toma de decisiones y hacerlos partícipes del cuidado(4). Sin embargo, algunos profesionales de Enfermería debido a falta de preparación suficiente y a experiencias negativas personales sobre la muerte, no se ven preparados para afrontar con eficiencia unos cuidados paliativos (5).

Por otro lado, en determinadas circunstancias, poder decidir dónde y cómo morir (voluntades anticipadas) es un derecho del ser humano (6). La toma de decisiones se debe apoyar en los códigos éticos y en el marco jurídico vigente. El Convenio del Consejo de Europa, relativo a los Derechos Humanos y a la Biomedicina, señala: "serán tomados en consideración los deseos expresados anteriormente con respecto a una intervención médica por un paciente, que, en el momento de la intervención, no se encuentre en situación de expresar su voluntad" (7).

\section{ANTECEDENTES}

La muerte es un proceso biológico y psicosocial. Las personas conviven con el miedo a la muerte, siendo uno de los principales temores y tratándolo como algo negativo. Los profesionales sanitarios están continuamente viviendo con la situación de muerte de otras personas, en contacto continuo con ella. Esto puede influir de manera negativa $o$ positiva en su actitud y manera de afrontarla (8).

Esta situación puede afectar al personal de Enfermería a nivel de calidad de vida laboral, numerosos estudios 
asocian la principal causa generadora de estrés en el personal de Enfermería está relacionada con la muerte y la agonía de los pacientes, siendo muchos profesionales incapaces de afrontar estas situaciones ${ }^{(9)}$.

La expectativa más importante de la profesión de Enfermería es preservar la vida y evitar el sufrimiento a los pacientes. La gran responsabilidad sobre la salud, la vida y el cuidado de otras personas, junto con el trato diario con los pacientes, son principales para la presencia de estrés y sus consecuencias (10).

Es necesario destacar también otros estresores de Enfermería como los conflictos con/entre familiares y pacientes, el estrés emocional con el cuidado del paciente crítico, la insatisfacción con el trabajo y las situaciones urgentes y graves, entre otros (11).

Los enfermeros más ansiosos y/o depresivos son aquellos a los que más le estresan el "contacto con el dolor y la muerte" (12).

Las Unidades de Cuidados Paliativos (UCl) son aquellas donde se encuentra el mayor porcentaje de grado de estrés en el personal sanitario, caracterizado por falta de educación relacionada sobre la muerte, conflicto laboral y contacto con el dolor y la muerte. Ya que, en la $\mathrm{UCl}$, las decisiones a menudo son irreversibles y comprometen la vida del paciente(13).

La falta de educación continuada al profesional sanitario genera síntomas como el estrés y la ansiedad para los profesionales de la $\mathrm{UCl}$, afectando más a mujeres y a titulares, comportándose como un estresor moderado (14).

\section{OBJETIVO}

Analizar y describir las experiencias de los profesionales de Enfermería respecto a la muerte en el ámbito personal y laboral.

\section{METODOLOGÍA}

Diseño: Estudio cualitativo basado en la fenomenología hermenéutica con marco epistemológico y metodológico de Gadamer, quién demandaba que al mirar al mundo de la vida hacemos un proceso circular en el que ponemos a prueba nuestra pre compresión del fenómeno, para así llegar a la fusión de horizontes entre el intérprete y los sujetos (15).

Participantes: La población escogida para nuestro estudio fueron 7 enfermeros con experiencia sobre la muerte, una enfermera especialista en cuidados paliativos y una enfermera matrona. Como criterios de inclusión encontramos: enfermeros de una institución privada de 
una ciudad de Andalucía, enfermeros presentes el día de la entrevista en la sala y enfermeros que firmaron el consentimiento informado. Como criterios de exclusión encontramos: enfermeros que trabajan allí, pero se negaron a realizar el estudio, y aquellos que no se encontraban allí el día de la entrevista. La muestra la forman aquellos enfermeros que firmaron el consentimiento informado, aceptaron participar en el estudio y se encontraban allí esa tarde, formados por 7 enfermeros más una enfermera especialista en cuidados paliativos. (Tabla 1)

Recogida de la información: Los participantes fueron entrevistados en la sala de reuniones de una institución privada de Almería. Las entrevistas se realizaron en diciembre de 2018. Los participantes firmaron el consentimiento escrito y completaron una breve encuesta demográfica. Cada entrevista semi-estructurada contaba con una batería de preguntas acerca de las percepciones respecto a la muerte social. Las entrevistas fueron llevadas a cabo por dos entrevistadores, con una duración aproximada de 20 minutos (entrevista sobre un tema concreto) y 45 minutos (grupo focal).
El grado de intervención en el texto respecto a la transcripción fue de 3 minutos por investigador. Una vez realizada la transcripción, fueron ingresadas en el programa informático ATLAS.ti versión 7.0, un programa informático que realiza la codificación de los datos recogidos. Posteriormente, categorizamos, es decir, ordenamos los significados formulados. Por consiguiente, incorporamos los resultados obtenidos a la descripción del fenómeno, y, para terminar, validamos los hallazgos obtenidos con los participantes.

\section{Análisis de los datos}

Las entrevistas y las notas de campo fueron transcritas textualmente. 
Aspectos éticos: En el desarrollo del proceso de investigación, para garantizar la confidencialidad y la legalidad de los procedimientos de obtención de datos, se ha obrado de acuerdo a la Ley Orgánica 15/1999 de 13 de diciembre de Protección de Datos de Carácter Personal, Real Decreto 1720/2007, de 21 de diciembre, por el que se aprobó el Reglamento de desarrollo de la Ley Orgánica $15 / 1999$, de 13 de diciembre, de protección de datos de carácter personal (BOE 17, de 19 de enero de 2008, págs. 4103-4136), la Declaración de Helsinki (1964) y las revisiones posteriores correspondientes, el informe Belmont (1978), Código de Nuremberg (1946) y el Convenio de Oviedo (1997), todos contienen los principios básicos que deben ser respetados en la efectuación de la investigación con seres humanos. Los investigadores se comprometieron a garantizar la confidencialidad y anonimato de los participantes, así como de los datos obtenidos.

Todos los datos que aquí aparecen han sido autorizados libremente a su publicación por parte de los participantes. En cuanto al conflicto de intereses planteado en esta investigación, fue de tipo personal, ya que el fin de dicho estudio fue aportar nuevos conocimientos acerca de este tema.
Rigor: Se ha realizado una triangulación de los datos mediante el empleo de diferentes estrategias, fuentes de información y métodos, hasta alcanzar la saturación de los datos. La validación del estudio presente se ha respaldado mediante la aprobación del hológrafo por parte de los participantes.

\section{RESULTADOS}

Del análisis de los datos emergen dos temas principales, que caracterizan las percepciones de la muerte social desde el punto de vista de los profesionales de Enfermería. (Figura 1)

\section{Tema 1. Afrontamiento social}

\section{de la muerte}

En esta categoría se refiere al afrontamiento social de la muerte, como se posiciona el personal de Enfermería ante el proceso de la muerte y la muerte en sí misma. Emergen dos modos de afrontamiento social de la muerte: trabajo interno para integrar la muerte como algo natural, y en el lado opuesto, dejarse llevar por una cultura de ocultación y obstinamiento, como un tema tabú y basado en el silencio al otro. Dentro de esta categoría, emergen dos subcategorías (Figura 2) 


\section{Subcategoría 1. Trabajo interno} para integrar la muerte en la existencia

Como personas, como profesionales y como sociedad, debemos hacer un trabajo reflexivo, un esfuerzo por integrar la muerte como un dato más de la existencia humana. Pensar la muerte, no ocultarla, incluir a los niños en sus rituales y proceso, formaría parte, según los participantes, de un proceso de afrontamiento social exitoso, destinado a ahorrar sufrimiento en la fase final de la vida.

Es un trabajo interno de cada persona, porque como dice ... a veces se nos olvida que un día de estos se nos va a acabar la vida, pensamos que vamos a ser inmortales, pero cuando nos llega es que como que nos viene de sorpresa cuando desde que naces sabes que vas a morir en un momento (GF-1).

Una de las cuestiones destacadas por los participantes para lograr esa integración es convivir con la palabra muerte, no evitarla, ni usar eufemismos para referirnos a ella.

Yo creo que es hasta necesario convivir con la palabra muerte, no que estés pensando todos los días me voy a morir, me voy a morir, pero sí saber que va a llegar y que es algo natural, y que ya está, que no pasa nada (GF-1).

Los entrevistados señalan no dejar elementos pendientes para sus fami- liares, además de cumplir con los objetivos y expectativas de su vida. También señalan haber vivido una vida plena.

$Y$ haber podido despedirse de la gente que es importante, no dejar problemas para ellos (GF-2)

\section{Subtema 2. Cultura de oculta} ción y obstinamiento

La sociedad occidental está basada en una cultura basada en el silencio y en la ocultación sobre temas relacionados con la muerte, más aún si cabe se suele hacer como protección alejar a los niños o a los menores de edad sobre este tema, recalcando la muerte como algo negativo y un tema tabú. También, el dar falsas esperanzas tanto a los enfermos como a los familiares supone una falta de comunicación y recalca los sentimientos negativos hacia la muerte.

Pero que también esa educación va variando porque por ejemplo cuando era pequeña a mí me alejaban de todas las personas, de todos los fallecimientos que había a mí me alejaban, me era como algo ajeno a mí. (GF-2).

Se ha intentado que los niños pe queños no vayan a los cementerios cuando tienen una pérdida cercana. (GF4) 
Como familiares, ante la pérdida de un ser querido, proyectamos nuestros miedos sobre la muerte.

Claro. Le estás ocultando una verdad que sabe a voces, pero no queremos ponerle la palabra porque nosotros mismos tenemos miedo a tener que enfrentarnos a esa situación. (GR-1)

Ocultamos a los pacientes y nuestros propios familiares las malas noticias, a pesar de ser ellos conscientes de la situación.

Sabes que a tu alrededor están hablando de cosas. Tú sabes cómo te sientes y muchas veces cuándo te encuentras solo. Llega la enfermera y le preguntas: 'Voy a morir, ¿verdad? ' (GF1)

\section{Categoría 2. La muerte como} proceso final de la vida

En esta segunda categoría los participantes se refieren al proceso de morir, tanto el proceso por el que pasa la persona que muere como la prestación de los cuidados que se requieren por parte del personal sanitario durante el mismo. Esta categoría tiene dos subcategorías que se representan en la Figura 3.

\section{Subcategoría 1. Rol de los pro-} fesionales de la salud

El papel de los profesionales de la sanidad radica en la importancia de estos en los momentos señalados, como es la muerte. Tienen un papel fundamental en cuanto a decir la verdad, no dar falsas esperanzas ni al enfermo ni a la familia y trabajar con ellos en un abordaje completo del tema. No es una solución ocultar a la familia el tema de la muerte, realmente hace falta una educación de los profesionales de la salud en cuanto a temas relacionados con la muerte, ya que muchos la ocultan o dejan su tema a otros profesionales de la salud.

Creo que Enfermería aparte de acompañar, tendría que informar y trabajar junto a la familia, que el paciente decida lo que quiera hacer. (GF-5)

Sin embargo, la falta de tiempo del personal de Enfermería y la falta de interés por querer abordar el tema de la muerte es clave para conocer si el paciente desea o no saber su pronóstico.

Por eso necesitas el tiempo, cuando has hablado un tiempo con esa persona por su manera de hablar o de callarse o de esquivar, de saber para saber si el paciente quiere saber o no. (GF5)

Los participantes señalan la falta de intimidad que existe para sobrellevar 
y afrontar el duelo ante la pérdida de un ser querido.

Un paciente que se muere a las 11 no puede tener intimidad en todo el tránsito de una planta, no hay intimidad, no hay nada. (E-1)

\section{Subcategoría 2. Conflicto en la} toma de decisiones.

El paciente debe ser el que tenga la palabra final, la falta de autonomía es el principal problema en el conflicto de toma de decisiones. La última palabra es parte del pronóstico de vida del paciente, dando así las voluntades anticipadas, generando todo esto un conflicto familiar entre el enfermo y la propia familia.

El paciente estaba sumamente mal, estaba muriéndose. En ese momento pedía morirse, pedía descansar, y la familia insistía al médico diciéndole "Por favor, haz algo". (GF-2)

La falta de existencia de un testimonio vital y de las voluntades anticipadas hace que los profesionales de la salud prioricen las peticiones de los familiares ante su familiar.

Pero ahí también está el trabajo de Enfermería en incidir que la gente... Hay mucha gente que no conoce las voluntades vitales anticipadas. Ahí está nuestro trabajo en difundir eso... esa información. (GF-1)

\section{DISCUSIÓN}

Norbert Elías centra su atención en el fenómeno de la muerte tal como occidente la enfrenta, divinizándola. En nuestro estudio no se ha dado esto, ya que nuestros participantes, enfermeros, se han centrado en la entrevista sobre su práctica clínica asistencial (16).

Los participantes hablaron de cultura de ocultación como forma social de afrontar la muerte. Otros autores han señalado esa manera de ocultación de muerte también en la vida cotidiana, como la proliferación de funerarias donde se evita la visión del cuerpo.

Todo esto representa una dificultad para integrar la muerte como un proceso natural, favoreciendo la falta de reflexión sobre la muerte en sí misma (17). A lo largo de los siglos podemos ver que el concepto negativo de la muerte perdura, ya que conforme se avanza en el tiempo la idea de la muerte se convierte cada vez más en un tema tabú y negativo. Algunos autores por consiguiente señalan la justificación de salvar vidas porque la muerte es mala, posponiéndola ya que una muerte temprana es peor que una muerte tardía (18).

El cuidado del entorno en la etapa final de la vida de dicho paciente es fundamental para garantizar dignidad en 
el proceso y poder ofrecerle intimidad que necesita (19). La muerte es considerada ya sea por parte del paciente como de los familiares, como el alivio del sufrimiento. Los entrevistados señalan no tener los conocimientos suficientes para dar malas noticias como es el tema de la muerte, aunque algunos autores señalan la escasa formación del profesional sanitario en estos temas (20).

Los profesionales de la salud entrevistados muestran la muerte como una etapa más de la vida, especialmente aquellos sanitarios que se encargan a diario del cuidado de personas ancianas (21).

Las entrevistas realizadas al personal de Enfermería nos permiten conocer historias personales y emotivas acerca de las experiencias cercanas a la muerte vividas por los enfermeros durante la práctica de su trabajo diario. Todo esto posibilita crear estrategias para mejorar la calidad de vida de este colectivo $\mathrm{y}$, asimismo, de los pacientes (22).

Los profesionales entrevistados destacan la importancia de trabajar con la familia para mejorar el afrontamiento de la muerte. En la actualidad, se ha institucionalizado la muerte ya sea en el ámbito público o privado, haciendo al personal sanitario partícipe de este proceso, recalcando que se prefiere una muerte en un hospital más que en el hogar (23).

Todo esto nos genera nuevas preguntas y problemas. La propuesta de un cuidado para prestar la mejor atención posible al paciente terminal y a sus familiares radica en la importancia de una formación específica en aspectos vinculados a la muerte, el duelo, la enfermedad terminal y cuidados paliativos. Por ello, muchos profesionales de Enfermería no se ven preparados para abordar el tema de la muerte, requiriendo éstos apoyo para proporcionar cuidado a estos pacientes (24).

Se esperaba que los participantes hablaran de los diferentes escenarios donde la muerte los hubiera sorprendido, tanto en el ámbito hospitalario como en el domicilio, pero no emergió en las entrevistas como en otras investigaciones publicadas (25).

Se recomienda el empleo de voluntades anticipadas por parte de los pacientes, donde expresan el deseo de cómo morir; de esta forma se garantiza el cumplimiento del derecho de autonomía (26). 


\section{CONCLUSIONES}

El afrontamiento de la muerte desde un punto de vista social puede resultar como algo positivo (trabajo interno de cada individuo) o como algo negativo (tratándolo como tema tabú y obstinamiento).

Los profesionales sanitarios juegan un papel fundamental para los propios pacientes y sus familiares, haciéndose más evidente en el tema de la muerte. No obstante, este tema sigue siendo ocultado por muchos profesionales bajo un manto del silencio. El personal de Enfermería es el indicado para hacer frente a las situaciones relacionadas con la muerte. La mayoría de estos profesionales sanitarios, reclaman desde su posición la falta de educación sobre el tema de la muerte, siendo muchos de ellos incapaces de afrontar situaciones relacionadas con la misma.

El personal de Enfermería debe ser capaz de proporcionar unos cuidados íntegros tanto a familiares como a los propios pacientes en las etapas finales de la vida, sin distinción de edad. Además, el paciente debe de tener la última palabra, aplicando así las voluntades anticipadas, otorgándole a él mismo la capacidad de tomar sus propias decisiones y ejerciendo su derecho a la autonomía. Esto último debe ser un punto clave, dado que podría deshacer un hecho que se produce con relativa frecuencia, como es el conflicto entre los deseos de los familiares y pacientes.

Este estudio puede suponer un primer paso para seguir estudiando y profundizar en las percepciones que tienen de las situaciones relacionadas con la muerte los profesionales sanitarios, pudiendo desarrollar técnicas y formas de actuar ante la muerte, favorables para los pacientes y su entorno y para los propios profesionales. 


\section{BIBLIOGRAFÍA}

1. Cevik, B, Kav, S. Attitudes and experiences of nurses toward death and caring for dying patients in Turkey. Cancer Nursing. 2013; 36(6), E58-65.

2. Dy, SM. Measuring the quality of palliative care and supportive oncology: principles and practice. $\mathrm{J}$ Support Oncol. 2013; 11(4), 160-164.

3. Miller, SC, Lima, JC, Intrator, O, Martin, E, Bull, J, Hanson, LC. Palliative Care Consultations in Nursing Homes and Reductions in Acute Care Use and Potentially Burdensome End-of-Life Transitions. Journal of the American Geriatrics Society. 2016; 64(11), 2280-2287.

4. Kohler, G, Sampalli, T, Ryer, A, Porter, J, Wood, L, Bedford, $L$, et al. Bringing value-based perspectives to care: including patient and family members in decision-making processes. International Journal of Health Policy and Management. 2017; 6(11), 661.

5. Peters, L, Cant, R, Payne, S, O'Connor, M, McDermott, F, Hood, K, et al. How death anxiety impacts nurses' caring for patients at the end of life: a review of literature. The open nursing journal. 2013; 7, 14.

6. Faden, RR, Beauchamp, TI, Kass, NE. Informed consent, comparative effectiveness, and learning health care. N Engl J Med. 2014; 370(8), 766-768.

7. WHO definition of palliative care (consultado 2 Enero de 2019). Disponible en: http://www.who.int/ cancer/palliative/definition/en/\#

8. Pascual, FM. Anxiety of nursing staff in the face of death in critical care units and its relationship with the patients' age. Enfermería intensive. 2011; 22(3), 96.

9. Anderson, NE, Kent, B, Owens, RG. Experiencing patient death in clinical practice: nurses' recollections of their earliest memorable patient death. International Journal of Nursing Studies. 2015; 52(3), 695-704.

10. Braun, M, Gordon, D, Uziely, B. Associations between oncology nurses' attitudes toward death and caring for dying patients. Oncology Nursing Forum. 2010; 37(1), E43-49.

11. Barth, AA, Weigel, BD, Dummer, CD, Machado, $\mathrm{KC}$, Tisott, TM. Stressors in the relatives of patients admitted to an intensive care unit. Revista Brasileira de terapia intensiva. 2016; 28(3), 323-329.

12. Gama, G, Barbosa, F, Vieira, M. Factors influencing nurses' attitudes toward death. International Journal of Palliative Nursing. 2012; 18(6), 267-273.

13. Terakado, A, Matsushima, E. Work stress among nurses engaged in palliative care on general wards. Psycho-Oncology. 2015; 24(1), 63-69.

14. Muñoz, ES, González, AIG, Alcaide, APR. ca 2-291: La falta de educación continuada como estresor laboral para enfermería de uci. Enfermería docente. 2016;1(106), 146.

15. Fernández Sola, C. Afrontar la muerte en ciencias de la salud [tesis]. Universidad de Almería; 2012.
16. Elías, N. La soledad de los moribundos. Fondo de Cultura Económica. 1th ed. Madrid; 1987.

17. Howarth, A. (2011) Concepts and Controversies In Grief And Loss. Journal Of Mental Health Counselin. 2011; 33 (1), 4-10.

18. Tollef, CS, Gamlund, E. The badness of death and priorities in health. BMC medical ethics. 2016; 17(1), 21.

19. Fascioli, A. Los cuidados paliativos al final de la vida: Expresión del reconocimiento del otro. Enfermería: Cuidados Humanizados. 2016; 5(2), 46-53.

20. Marti-Garcia, C, Garcia-Caro, MP, Schmidt-Riovalle, J, Fernández-Alcántara, $M$, Montoya-Juárez, R, Cruz-Quintana, F. Formación en cuidados paliativos y efecto en la evaluación emocional de imágenes de muerte. Medicina Paliativa. 2016; 23(2), 72-78.

21. Costa, CR, Costa, LPD, Aguiar, N. The role of nursing the patient with brain death in the ICU. Revista Bioética. 2016;24(2), 368-373.

22. Velarde, JFG, Luengo, RG, González, RH, Cardenete, CR, Alvarado, GZ, Palacios, DC. Facing death in the intensive care unit. A phenomenological study of nurses' experiences. Contemporary nurse. 2016; 52(1), 1-12.

23. Morais, IMD, Nunes, R, Cavalcanti, T, Soares, AKS, Gouveia, VV. Perception of "dignified death" for students and doctors. Revista Bioética. 2016;24(1), 108-117.

24. Delgado, DS, Juarez, RV, Pellicer, BG, Redondo, LC, Ramón, EA, López, IM, et al. Nursing action before the terminal patient physical care. Revista de enfermeria (Barcelona, Spain). 2014; 37(11), 26-29.

25. Guerrero, MV. Aportando valor al cuidado en la etapa final de la cronicidad. Enfermería clínica. 2014;24(1), 74-78. 


\section{ANEXOS Y TABLAS}

Figura 1. Temas, subcategoría y unidades de significado. Fuente: elaboración propia.

\begin{tabular}{|c|c|c|}
\hline TEMA & SUBCATEGORIAA & UNIDADES DE SIGNIFICADO \\
\hline \multirow{39}{*}{$\begin{array}{l}\text { Afrontamiento social } \\
\text { de la muerte }\end{array}$} & \multirow[t]{11}{*}{ Trabajo interno para integrar la muerte } & Evitar sufrimiento a los seres queridos \\
\hline & & Morir tras una vida cumplida \\
\hline & & Pensar en la muerte \\
\hline & & Muerte como alivio del sufrimiento \\
\hline & & Naturalizar la muerte \\
\hline & & No querer ser una carga \\
\hline & & Despedirse de los seres queridos \\
\hline & & Informar a los niños \\
\hline & & Convivir con la muerte \\
\hline & & Muerte como proceso natural \\
\hline & & Socializar la muerte \\
\hline & \multirow{8}{*}{$\begin{array}{l}\text { Cultura de la ocultación y } \quad \text { del } \\
\text { obstinamiento }\end{array}$} & Alcjar a los niños \\
\hline & & Dar falsas esperanzas \\
\hline & & Miedo a la muerte \\
\hline & & Muerte como algo negativo \\
\hline & & Muerte como fracaso de la ciencia \\
\hline & & Ocultar pronóstico \\
\hline & & Tabú \\
\hline & & Vivir como inmortales \\
\hline & \multirow[t]{5}{*}{ Conflicto en la toma } & Conflicto familiar \\
\hline & & Falta de autonomía \\
\hline & & Objeción de la conciencia \\
\hline & & Obstinamiento familiar \\
\hline & & Voluntades anticipadas \\
\hline & \multirow[t]{15}{*}{ Rol de los profesionales de la salud } & Anticipar la muerte \\
\hline & & Crear la oportunidad \\
\hline & & Cuidar el entorno \\
\hline & & Dar falsas esperanzas \\
\hline & & Despersonalización \\
\hline & & Dilemas éticos \\
\hline & & Empatia \\
\hline & & Falta de recursos en la comunicación \\
\hline & & No sacar el tema de la muerte \\
\hline & & Objeción de la conciencia \\
\hline & & Obstinamiento terapéutico \\
\hline & & Ocultar pronóstico \\
\hline & & Presencia \\
\hline & & Tecnificación de la asistencia \\
\hline & & Trabajar con la familia \\
\hline
\end{tabular}


PERCEPCIONES DE LOS PROFESIONALES DE ENFERMERÍA SOBRE LA MUERTE

Figura 2. Mapa conceptual de la categoría "Afrontamiento social de la muerte".

Fuente: Elaboración propia.

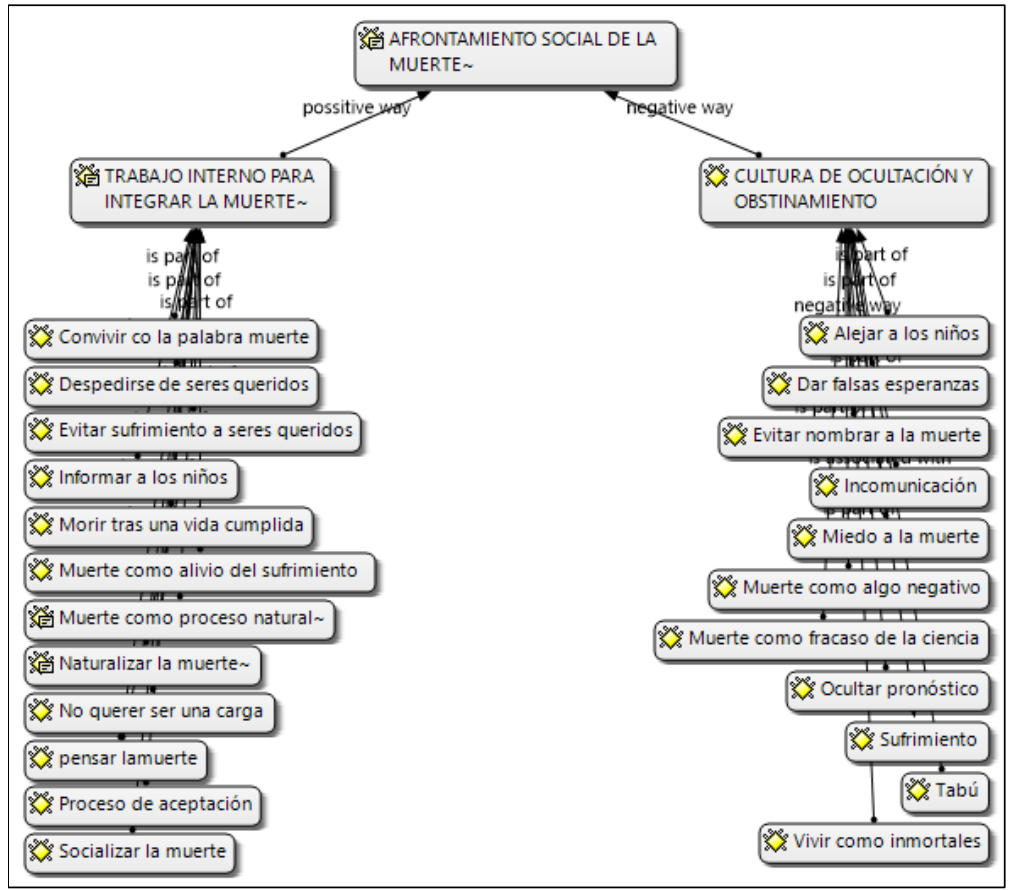

Figura 3. Mapa conceptual de la categoría "La muerte como proceso final de la vida". Fuente: Elaboración propia.

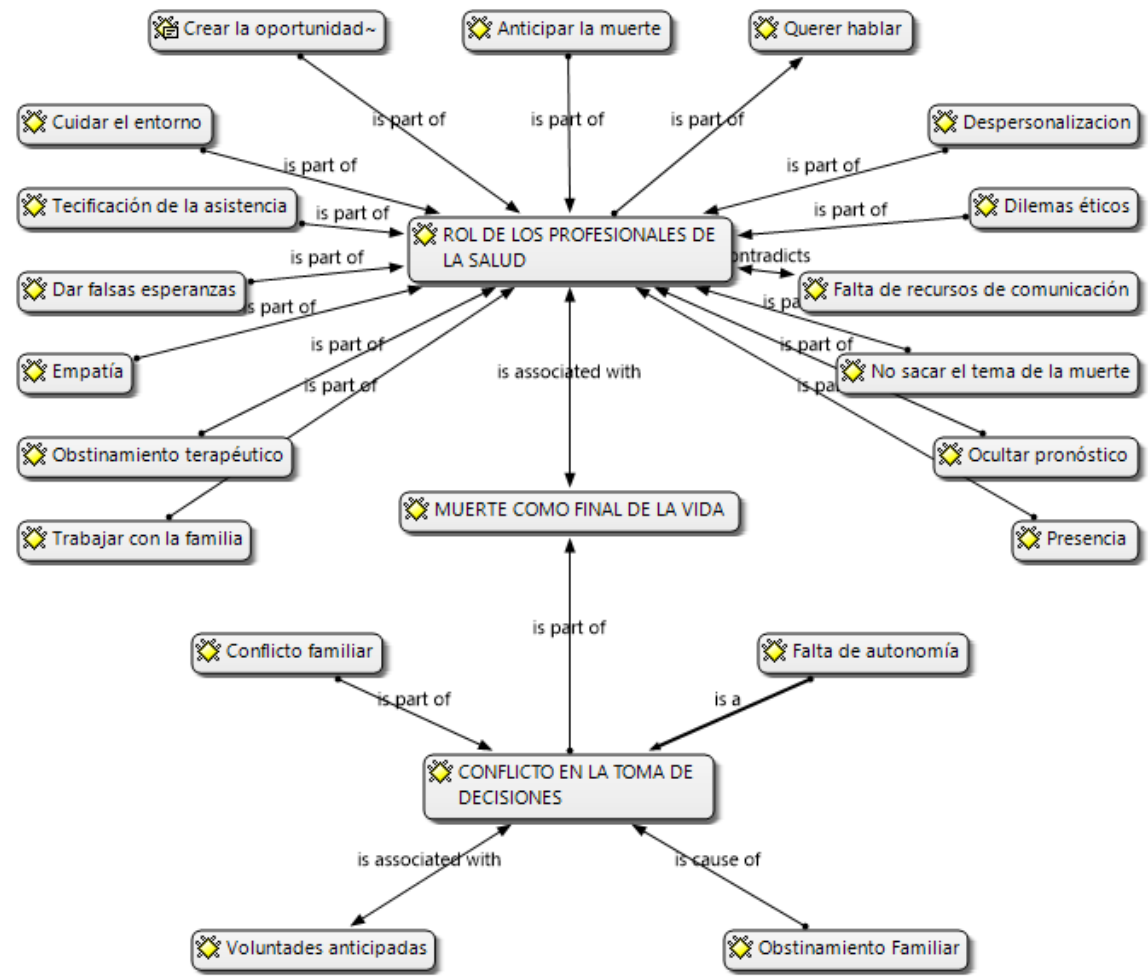


PERCEPCIONES DE LOS PROFESIONALES DE ENFERMERÍA SOBRE LA MUERTE

Tabla 1. Datos socio-demográficos de los participantes. La entrevista en profundidad surgió de uno de los participantes del grupo focal. Fuente: Elaboración propia.

\begin{tabular}{|l|l|l|l|l|l|}
\hline & $\begin{array}{l}\text { Sexo } \\
\text { Masculino }\end{array}$ & $\begin{array}{l}\text { Sexo } \\
\text { Femenino }\end{array}$ & $\begin{array}{l}\text { Profesión: } \\
\text { enfermero }\end{array}$ & $\begin{array}{l}\text { Edad } \\
\text { (años) }\end{array}$ & $\begin{array}{l}\text { Experiencia } \\
\text { (años) }\end{array}$ \\
\hline $\begin{array}{l}\text { Entrevista en } \\
\text { profundidad } \\
(n=1)\end{array}$ & 0 & 1 & 1 & 23 & 2 \\
\hline $\begin{array}{l}\text { Grupo focal } \\
(n=8)\end{array}$ & 3 & 5 & 8 & $22-50$ & $0-25$ \\
\hline
\end{tabular}

